\title{
Antitumor effect of photodynamic therapy with a novel targeted photosensitizer on cervical carcinoma
}

\author{
PENG-XI LI ${ }^{1}$, JIANG-HONG MU ${ }^{2}$, HUA-LANG XIAO ${ }^{2}$ and DONG-HONG LI ${ }^{1}$ \\ ${ }^{1}$ State Key Laboratory of Trauma, Burn and Combined Injury, The Second Department of Research Institute of Surgery, \\ ${ }^{2}$ Department of Pathology, Daping Hospital, Third Military Medical University, Chongqing, Sichuan 400042, P.R. China
}

Received June 25, 2014; Accepted September 19, 2014

DOI: 10.3892/or.2014.3593

\begin{abstract}
The antitumor effect of photodynamic therapy (PDT) mediated by a novel photosensitizer I (Ps I; $\{\gamma$-[N-poly (ethyleneglycol)]folic acid\}-5,10,15-tris(3-hydroxyphenyl)-20(4-carboxyphenyl)chlorin), in which chlorin was used as a photoactive unit, folic acid as a tumor-targeting warhead, and polyethylene glycol as a linker, on cervical carcinoma was studied in vitro and in vivo. Ps I exhibited a considerably higher cellular uptake by HeLa cells than folic acid-free analogue Ps A (tert-butyl N-poly(ethyleneglycol)ethylcarbamate-5,10,15-tris(3hydroxyphenyl)-20-(4-carboxyphenyl)chlorin), and the cellular uptake by HeLa cells of Ps I could be competitively inhibited by excess folic acid. Moreover, at different time points after the intravenous (i.v.) injection of Ps I and A, Ps I produced a >2-fold higher tumor to normal tissue ratio in tumor-bearing nude mice as compared to Ps A. MTT assay indicated that the HeLa cell proliferation inhibition ratio was increased 34\% after Ps I-PDT compared with Ps A-PDT with a photosensitizer concentration of $15.2 \mu \mathrm{mol} / \mathrm{l}$. Administration of Ps I ( $7 \mathrm{mg} / \mathrm{kg}$, i.v.) followed by light exposure $\left(80 \mathrm{~J} / \mathrm{cm}^{2}\right)$ markedly suppressed the growth of xenograft tumors, and the tumor volume was 10-fold smaller than that of the control group. Tumor growth inhibition in vitro and in vivo had an obvious dependency on the Ps I concentration and irradiation dose. The mode of cell death post-Ps I-PDT was analyzed by flow cytometry, confocal laser scanning microscopy, and electron microscope, and the results suggested that apoptosis was the primary mode of HeLa cell death induced by Ps I-PDT. The results also demonstrated that tumor targeting of Ps I was clearly improved because of the endocytosis mediated by the folate receptor. As a result, Ps I-PDT exhibited higher antitumor activity than Ps A-PDT and has potential as an alternative treatment modality for cervical cancer.
\end{abstract}

Correspondence to: Professor Dong-Hong Li, State Key Laboratory of Trauma, Burn and Combined Injury, The Second Department of Research Institute of Surgery, Daping Hospital, Third Military Medical University, 10 Changjiang Road, Chongqing 400042, P.R. China E-mail: lqs_cq@aliyun.com

Key words: photosensitizer I, cervical cancer, tumor targeting, antitumor activity, apoptosis

\section{Introduction}

Cervical cancer is the second most common cancer and the third leading cause of mortality among women worldwide. Each year $\sim 500,000$ women worldwide develop cervical cancer, of which $>90 \%$ is found in developing countries (1). The demographics of cervical cancer patients have been shifting towards younger patients, with the majority of them being single or nullipara women (2). Thus, there is a strong demand to maintain their fertility function while effectively treating the cancer. In addition, an effective and conservative therapy method is also required for elderly patients who cannot undergo surgery or radiotherapy, as well as patietns who refuse surgery (3).

Photodynamic therapy (PDT) is emerging as a viable conservative treatment for various types of cancer. PDT is based on the light-induced activation of a photosensitizer that results in subsequent in situ production of reactive oxygen species (ROS), which directly destroy cells that have accumulated the photosensitizer. Thus, the photosensitizer, light and oxygen are three important elements to determine the efficiency of PDT $(4,5)$.

However, the application of most existing photosensitizers is limited because of their disadvantages, mainly including (6) i) a relatively low tumor selectivity, which can lead to undesired side effects in normal tissues, especially the prolonged cutaneous photosensitivity post-PDT and ii) lack of water solubility, which hampers the development of pharmaceutical formulations, inducing the aggregation of photosensitizer in the physiological medium, resulting in a severe reduction of the photochemical properties and the bioavailability of the photosensitizer (7-9). Thus, during the last few years, a great deal of effort has been invested into improving the targeting of photosensitizers by conjugating photosensitizers to tumor-seeking molecules, such as epidermal growth factor, monoclonal antibodies, carrier proteins, carbohydrates, and hydrophilic polymers (10-13).

Based on the fact that the folate receptor in human epithelial cancer cells was overexpressed, despite its absence from most normal cells and the high affinity between folic acid and the folate receptor $(14,15)$, a targeted photosensitizer (Ps I) was designed and generated in our laboratory using folic acid as a tumor-seeking bullet, polyethylene glycol (PEG) as the linker, and chlorin, an analogue of mTHPC (temoporfin), 
as a photoactive block (Fig. 1A). mTHPC is one of the most potent second-generation photosensitizers (16). PEG, when used as a linker, improves the hydrophilicity of the conjugate and prevents the recognition of the reticuloendothelial system (RES) of the conjugate (17). The results of previous studies indicated that this new photosensitizer exhibited much higher tumor targeting and water solubility but lower aggregation than that of its precursor, chlorin (18). The aim of the present study was to evaluate further the tumor targeting and photodynamic activity of PS I in cervical cancer in vitro and in vivo. In addition, the cell death mode induced by PS I-based PDT was investigated.

\section{Materials and methods}

Chemicals and reagents. Fetal bovine serum, penicillin/ streptomycin solution, and RPMI-1640 were purchased from HyClone (South Logan, UT, USA). Folate-free RPMI-1640 was purchased from Gibco (Grand Island, NY, USA). Trypsin, methylthiazolyldiphenyl-tetrazolium bromide (MTT), and dimethyl sulfoxide (DMSO) were purchased from SigmaAldrich (St. Louis, MO, USA). An Annexin V-PI Apoptosis Detection kit was purchased from BD Bioscience (San Jose, CA, USA). 4',6-Diamidino-2-phenylindole dihydrochloride (DAPI) and a BCA kit were purchased from Beyotime (Shanghai, China). A terminal deoxynucleotidyl transferasemediated deoxyuridinetriphosphate nick end-labeling (TUNEL)-FITC Apoptosis Detection kit was purchased from Invitrogen (Carlsbad, CA, USA). The photosensitizers (PS I and A), with a purity of $>98 \%$, were produced in our laboratory. Stock solutions of PS I and A $(1 \mathrm{mg} / \mathrm{ml})$ were prepared in FBS- and folate-free RPMI-1640 and stored in the dark at $4^{\circ} \mathrm{C}$.

Animals and cell lines. Four-week-old BALB/c nude mice, weighing 16-18 g, were obtained from HFK Bio-Technology Co., Ltd. (Beijing, China). The mice were kept in an air-conditioned facility fitted with an artificial $12 \mathrm{~h}$ light-dark cycle and provided standard food and filtered water. Experiments were conducted in compliance with the Animal Management Rules of the Ministry of Health of the People's Republic of China (document no. 55, 2001) and the guidelines for the Care and Use of Laboratory Animals of China Pharmaceutical University. The human cervical carcinoma HeLa cell line was obtained from the Chinese Academy of Science Shanghai Cell Library and was cultured in RPMI-1640 medium supplemented with $10 \% \mathrm{FBS}$, penicillin $(100 \mu / \mathrm{ml})$ and streptomycin $(100 \mu / \mathrm{ml})$ at $5 \% \mathrm{CO}_{2} 37^{\circ} \mathrm{C}$ in a humidified incubator.

Cellular uptake. HeLa cells were cultured with folate-free RPMI-1640 for $24 \mathrm{~h}$. After exposure to the photosensitizer for $24 \mathrm{~h}$, the cells were washed with PBS and fixed with $3.7 \%$ paraformaldehyde for $15 \mathrm{~min}$. The fluorescence intensity of the photosensitizers in the cells was analyzed by confocal laser scanning microscopy (Ex, $480 \mathrm{~nm} / \mathrm{Em}, 650 \mathrm{~nm})$. In addition, after the HeLa cells were exposed to $15.2 \mu \mathrm{mol} / 1$ of Ps I and A with or without folic acid $\left(2 \times 10^{3} \mu \mathrm{mol} / \mathrm{l}\right)$ for 2,8 or $24 \mathrm{~h}$, the cells were washed with PBS, and $500 \mu \mathrm{l}$ of $4 \%$ SDS was added to each well. The solutions were centrifuged for 9,000 rpm at $4^{\circ} \mathrm{C}$ for $20 \mathrm{~min}$, and the fluorescence intensity of the photosensitizers in the supernatant was measured using an F-4500 fluorescence spectrophotometer (Ex, $480 \mathrm{~nm}$ and Em, $650 \mathrm{~nm}$; Hitachi, Japan). In addition, the total protein concentration of each well was determined using the BCA kit according to the manufacturer's instructions. For each incubation time, the determination was run in triplicate.

Photocytotoxicity assay. The HeLa cells were inoculated into detachable 96-well culture plates and were incubated in Ps I or A for $24 \mathrm{~h}$ in the dark before exposure to the red light generated by a KDH150B red-light therapy instrument $(600-700 \mathrm{~nm}$; Kedian Co., Beijing, China). After incubation for another $24 \mathrm{~h}$ in the dark, the cells were incubated in $0.5 \mathrm{mg} / \mathrm{ml} \mathrm{MTT} \mathrm{solu-}$ tion for $4 \mathrm{~h}$, and the resulting formazan crystal was dissolved in DMSO. The absorbance at $492 \mathrm{~nm}$ was measured using a Microplate Reader Model 450 (Bio-Rad, Hercules, CA, USA). Cell viability was proportional to the $\mathrm{A}_{492}$ value and was expressed as a percentage of the cell viability of the untreated control cells. For each concentration or irradiation dose, three experiments were performed.

Detection of apoptotic characteristics of HeLa cells following PDT treatment. HeLa cells were incubated with $15.2 \mu \mathrm{mol} / 1$ of Ps I for $24 \mathrm{~h}$ and then exposed to red light at a $15 \mathrm{~J} / \mathrm{cm}^{2}$ irradiation dose. After $12 \mathrm{~h}$, the HeLa cells were harvested and double-stained using an Annexin V-PI apoptosis detection kit according to the manufacturer's instructions. The stained cells were analyzed using a FACSCalibur flow cytometer (BD Biosciences). At least 10,000 events were collected for each sample.

In addition, an aliquot of the HeLa cells (in the PDT and control groups) was fixed with $4 \%$ paraformaldehyde for $15 \mathrm{~min}$, incubated with $3 \%$ SDS for $3 \mathrm{~min}$ and then stained with $500 \mathrm{ng} / \mathrm{ml}$ DAPI for $5 \mathrm{~min}$. The cells were visualized under laser confocal microscopy [TCS sp5 (DAPI Ex, $358 \mathrm{~nm}$ and Em, $461 \mathrm{~nm}$ ); Leica, Buffalo Grove, IL, USA]. In addition, aliquots of the harvested HeLa cells (in the PDT and control groups) were fixed in $2.5 \%$ glutaraldehyde. After centrifugation at $1500 \mathrm{rpm}$ for $10 \mathrm{~min}$ and being washed with PBS, the cells were post-fixed in $1.0 \%$ osmium tetroxide, dehydrated, soaked and embedded in Epon 618 for subsequent sectioning. The ultrathin sections were stained with uranyl acetate and lead citrate, and examined under a transmission electron microscope (TECNAI 10; Phillips, The Netherlands).

Animal model. A xenograft tumor model was created by subcutaneous injection of HeLa cells $\left(\sim 2 \times 10^{6}\right.$ in $200 \mu$ l serum-free culture medium) in the right forelimb of nude mice. Tumor sizes and body weights were measured every 2 days. Tumor volume was calculated by a modified ellipsoidal formula: $(\mathrm{LxWx} \pi) / 6$ (19), where L was the length, and W the width. The animals were used for experiments when the tumors measured $\sim 6-8 \mathrm{~mm}$ in diameter, and there was no ulcer on the epidermis.

Tissue distribution of $P$ S I and A. The tissue distribution of Ps I and A were measured according to a previously established method with some modifications (20). Briefly, after the Ps I and A injection (at a dose of $5 \mathrm{mg} / \mathrm{kg}$ body weight) via the tail vein, the mice were randomly sacrificed, and the tissues of the brain, heart, liver, spleen, kidney, bladder, small intestine, muscle, skin and tumor were collected, washed, 

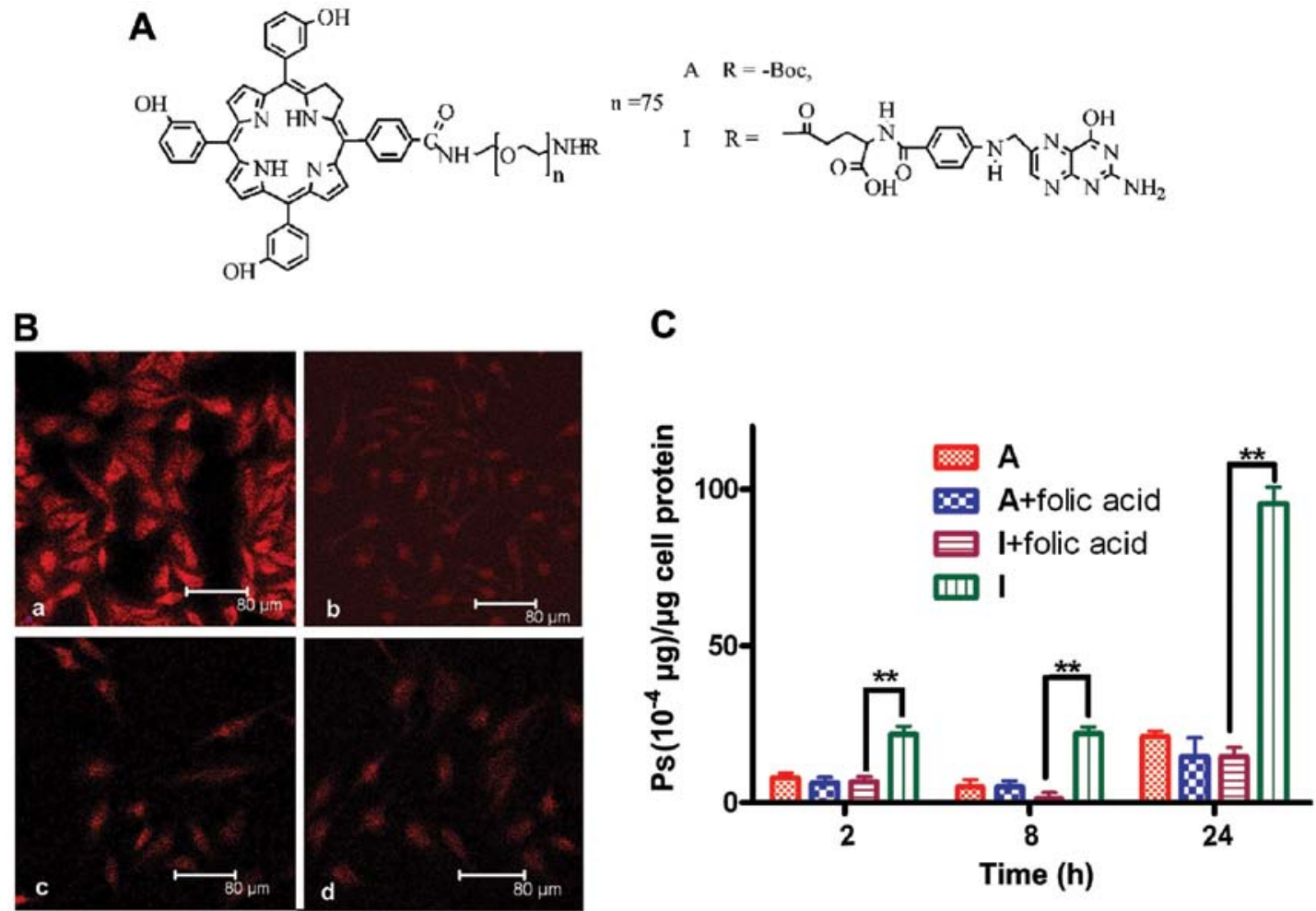

Figure 1. Uptake of photosensitizer I (Ps I). (A) Chemical structure of Ps I and A. (B) Confocal laser scanning microscopy of HeLa cells incubated with (a) $15.2 \mu \mathrm{mol} / 1$ of Ps I, (b) $15.2 \mu \mathrm{mol} / 1$ of Ps I $+2 \times 10^{3} \mu \mathrm{mol} / 1$ of folic acid, (c) $15.2 \mu \mathrm{mol} / 1$ of Ps A, (d) $15.2 \mu \mathrm{mol} / 1$ of Ps A $+2 \times 10^{3} \mu \mathrm{mol} / 1$ of folic acid for $24 \mathrm{~h}$. (C) Influence of incubation time on the cellular uptake of Ps I and A. The error bar means error S and $n=3,{ }^{* *} \mathrm{P}<0.01$.

weighed and homogenized in a mixture solution of $1 \mathrm{ml}$ of physiological saline and $0.5 \mathrm{ml}$ of methanol. After centrifugation for $15 \mathrm{~min}$ at $5,000 \mathrm{rpm}$, the fluorescence $(\lambda \mathrm{Ex}, 485 \mathrm{~nm}$ and $\lambda E m, 645 \mathrm{~nm}$ ) of the solubilized Ps in the supernatant was measured by a spectrofluorophotometer (Synergy HT; Bio-Tek, Winooski, VT, USA). The fluorescence intensity of the tissue samples was proportional to the content of Ps I and A in tissues. The tumor to normal tissue ratios were calculated by dividing the data of a.u./mg in the tumor by that measured in each individual organ. The background signal was subtracted after measuring the tissue autofluorescence of non-injected HeLa-bearing mice.

In vivo PDT efficacy. The mice were randomly distributed into the different therapy and control groups when the tumor grew to $\sim 6-8 \mathrm{~mm}$ in size. After the tail vein injection of Ps I at $6 \mathrm{~h}$, the tumor was illuminated with red light. The tumor volume was monitored every day for 14 days with vernier calipers. After the mice were sacrificed, the tumor was excised, measured and recorded as images. For further histological examination, the excised tumors, livers, spleens and kidneys were fixed in $3.7 \%$ formaldehyde in PBS, embedded in paraffin, and the sections were stained with hematoxylin and eosin and examined under a light microscope.

Apoptotic analysis of tumor sections. Cell apoptosis in the tumor tissue was analyzed first by a TUNEL assay using a TUNEL-FITC apoptosis detection kit according to the manufacturer's instructions. The excised tumors were subsequently fixed in $2.5 \%$ glutaraldehyde. After routine fixing, dehydration, soaking, embedding, sectioning and dyeing according to the TEM examination request, the ultramicrostructure of the tumor was observed under the microscope.

Statistical analysis. The results are expressed as the mean \pm standard deviation (SD). Statistical analysis was performed with SPSS version 16.0. An independent sample t-test was employed to compare the means between the two groups. $\mathrm{P}<0.05$ was considered to be statistically significant.

\section{Results}

Cellular uptake of Ps I. The cellular uptake of Ps I and A by HeLa cells was first investigated using laser scanning confocal microscopy. After incubation with Ps I, strong red fluorescence of Ps I was observed in the cytoplasm of HeLa cells (Fig. 1B-a), and the fluorescence intensity became weaker with the addition of excess free folic acid (Fig. 1B-b). However, for HeLa cells incubated with Ps A, a slight amount of Ps A aggregated on the cell surface (Fig. 1B-c). No visible differences between Fig. 1B-c and -d were observed.

The cellular uptake of Ps I and A by HeLa cells was quantitatively determined by fluorescence analysis. With the extension of incubation time, the cellular uptake of Ps I by HeLa cells increased, and, the disparity between cells with or without folic acid was more obvious (Fig. 1C). For example, when the incubation time was extended from 2 to $24 \mathrm{~h}$, the disparity of cellular uptake of Ps I with or without the presence of folic acid increased from 3.1- to 6.5-fold. However, the presence of folic acid did not affect the uptake of Ps A by HeLa cells, although the cellular uptake slightly increased with incubation time. 

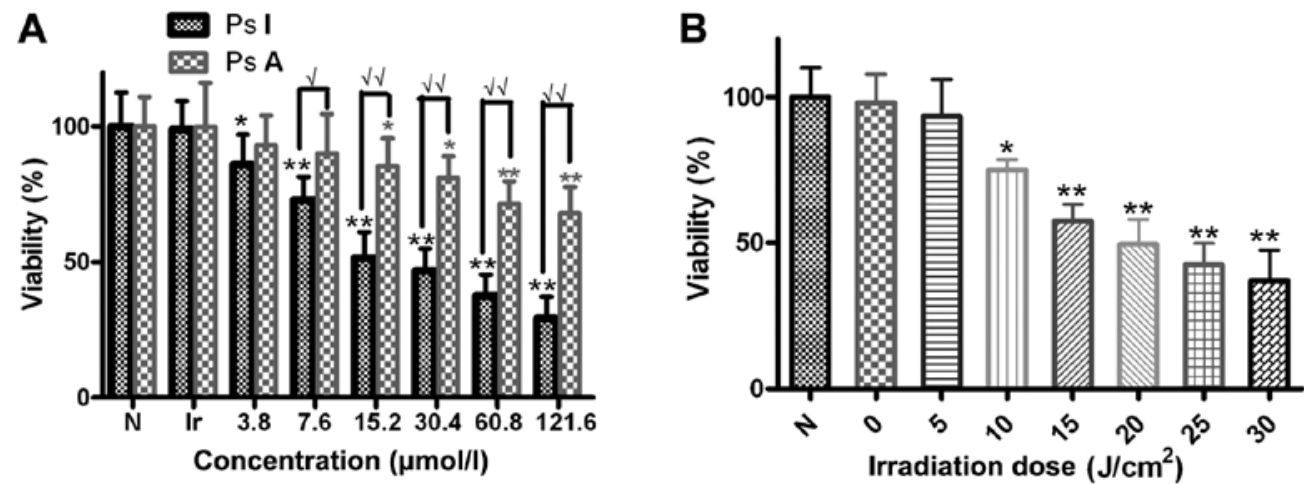

Figure 2. The viability of HeLa cells measured by MTT assay. (A) Concentration-dependent photocytotoxicity. HeLa cells were treated with photosensitizer I (Ps I) or A at the indicated concentration for $24 \mathrm{~h}$ and then irradiated with $15 \mathrm{~J} / \mathrm{cm}^{2}$ of red light. (B) Irradiation dose-dependent photocytotoxicity. HeLa cells were treated with $15.2 \mu \mathrm{mol} / 1$ of Ps I for $24 \mathrm{~h}$ and then exposed to the red light at the indicated dose. The error bar means error S and $\mathrm{n}=8$. Significant differences $\left({ }^{*} \mathrm{P}<0.05\right.$ and $\left.{ }^{* *} \mathrm{P}<0.01\right)$ versus the control groups. ${ }^{\sqrt{ }} \mathrm{P}<0.05$ and ${ }^{\sqrt{ }} \mathrm{P}<0.01$.

A

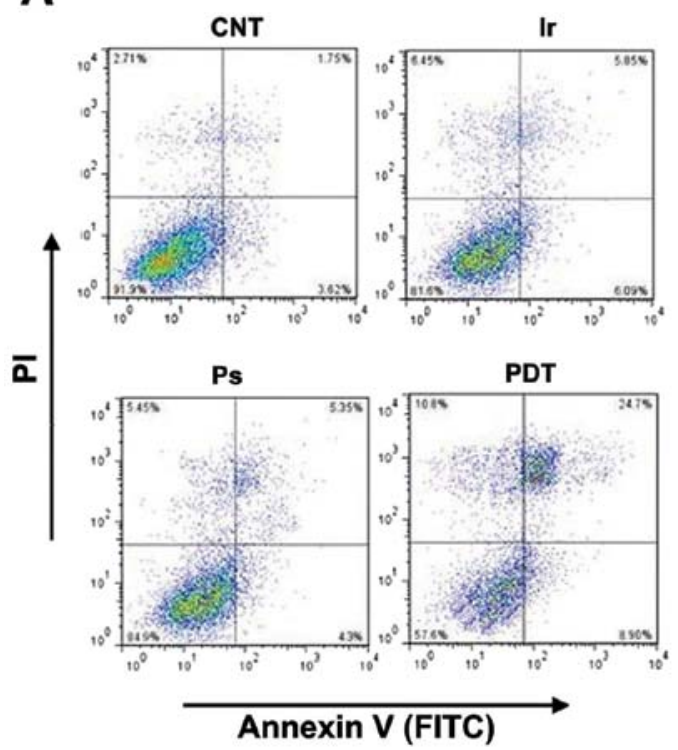

B

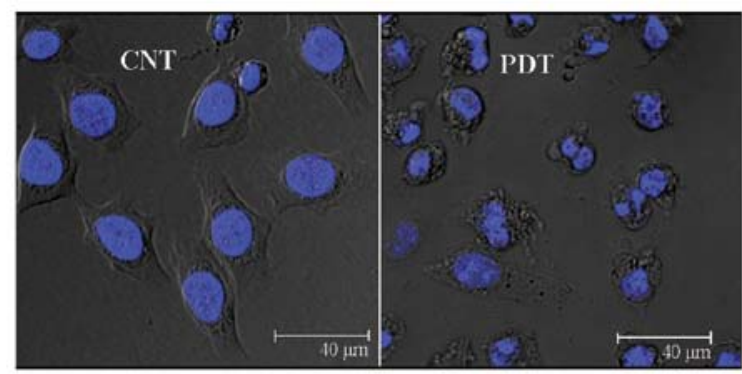

C

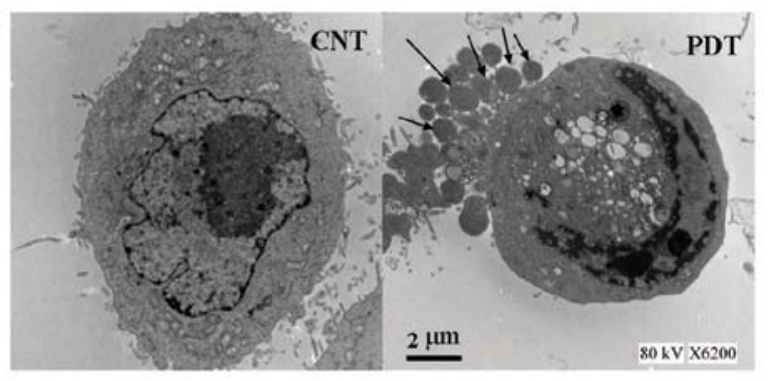

Figure 3. Apoptotic characteristics of HeLa cells after photosensitizer I (Ps I)-photodynamic therapy (PDT). (A) Results of flow cytometric analysis. CNT-untreated cells, Ir-only treated with red light, Ps-only incubated in Ps I, PDT-treated with Ps I, and then irradiated with red light. (B) The results of confocal laser scanning microscopy of HeLa cells stained by 4',6-diamidino-2-phenylindole dihydrochloride (DAPI). Scale bar, $40 \mu \mathrm{m}$. (C) Electron micrographs of HeLa cells. Scale bar, $2 \mu \mathrm{m}$.

Photodynamic activity of Ps I in vitro. The photodynamic activity of Ps I and Ps A in HeLa cells was measured by MTT assay. The viability of HeLa cells in the Ps I-PDT group was lower than that of cells in the Ps A-PDT group at all concentrations of photosensitizer tested in our assay (Fig. 2A). However, the cells illuminated without Ps I (Fig. 2A) and the cells kept in the dark in the presence of Ps I (Fig. 2B) did not present any significant loss of viability in our system. Additionally, the cytotoxicity induced by Ps I-PDT had a positive correlation with the concentration of Ps $I(R=0.763$ and $P=0.017)$ (Fig. 2A) and the irraditation dose $(\mathrm{R}=0.962$ and $\mathrm{P}=0.001)$ (Fig. 2B).

Apoptosis of HeLa cells after Ps I-PDT. The percentage of apoptotic and necrotic cells was first quantified via flow cytometric analysis. Compared with the control group, the number of apoptotic and necrotic cells only slightly increased in the irradiation alone group and the Ps I without light group.
However, the numbers significantly increased in the PDT group, especially the number of apoptotic cells (Fig. 3A). After PDT, cell rounding, shrinkage and deformation and typical nuclear fragmentation were observed (Fig. 3B). Under an electron microscope, the untreated control cells exhibited normal morphology. However, HeLa cells treated with Ps I-PDT displayed an apoptotic morphology, including the loss of microvilli and blebbing of the cell membrane, chromatin condensation around the nuclear membrane into dense halfmoon lumps, and the occurrence of representative apoptotic bodies alongside the HeLa cells with lobulated protuberance of cytoplasm (Fig. 3C, arrow).

Tissue distribution of Ps I and A. Ps I and A exhibited a different biodistribution in normal tissues, and the largest accumulations were found to occur in the kidney and liver, respectively, followed by the liver (or kidney) and intestine, 
Table I. Comparison of tumor to normal tissue ratios in various organs after intravenous administration of $5 \mathrm{mg} / \mathrm{kg}$ Ps A and I.

\begin{tabular}{|c|c|c|c|c|c|c|}
\hline Tissue & $0.5 \mathrm{~h}$ & $1 \mathrm{~h}$ & $3 \mathrm{~h}$ & $6 \mathrm{~h}$ & $12 \mathrm{~h}$ & $24 \mathrm{~h}$ \\
\hline \multicolumn{7}{|l|}{ Ps A } \\
\hline Heart & $0.71 \pm 0.23$ & $0.82 \pm 0.20$ & $0.77 \pm 0.13$ & $4.79 \pm 0.49$ & $10.67 \pm 1.13$ & $6.38 \pm 0.20$ \\
\hline Liver & $0.14 \pm 0.17$ & $0.21 \pm 0.07$ & $0.11 \pm 0.08$ & $0.32 \pm 0.05$ & $0.42 \pm 0.03$ & $0.50 \pm 0.08$ \\
\hline Spleen & $0.47 \pm 0.13$ & $0.49 \pm 0.08$ & $0.27 \pm 0.10$ & $1.57 \pm 0.43$ & $1.02 \pm 0.23$ & $1.42 \pm 0.33$ \\
\hline Kidney & $0.54 \pm 0.09$ & $0.30 \pm 0.06$ & $0.26 \pm 0.03$ & $0.31 \pm 0.04$ & $0.45 \pm 0.07$ & $0.56 \pm 0.04$ \\
\hline Intestine & $0.19 \pm 0.10$ & $0.29 \pm 0.12$ & $0.23 \pm 0.05$ & $0.59 \pm 0.23$ & $0.78 \pm 0.13$ & $0.86 \pm 0.07$ \\
\hline Bladder & $0.29 \pm 0.07$ & $0.49 \pm 0.11$ & $0.64 \pm 0.19$ & $1.36 \pm 0.25$ & $1.31 \pm 0.13$ & $2.55 \pm 0.14$ \\
\hline Muscle & $1.50 \pm 0.12$ & $2.25 \pm 0.20$ & $3.13 \pm 0.45$ & $4.55 \pm 0.41$ & $5.33 \pm 0.39$ & $2.83 \pm 0.09$ \\
\hline Skin & $0.83 \pm 0.10$ & $0.67 \pm 0.15$ & $0.55 \pm 0.16$ & $0.65 \pm 0.03$ & $0.59 \pm 0.07$ & $0.61 \pm 0.05$ \\
\hline \multicolumn{7}{|l|}{ Ps I } \\
\hline Heart & $2.22 \pm 0.37$ & $1.46 \pm 0.17$ & $4.10 \pm 0.37$ & $14.18 \pm 0.77$ & $20.50 \pm 1.63$ & $16.13 \pm 0.58$ \\
\hline Liver & $2.86 \pm 0.17$ & $1.07 \pm 0.07$ & $1.23 \pm 0.07$ & $1.54 \pm 0.17$ & $1.67 \pm 0.10$ & $2.69 \pm 0.23$ \\
\hline Spleen & $1.82 \pm 0.27$ & $1.17 \pm 0.13$ & $2.44 \pm 0.13$ & $5.24 \pm 0.23$ & $3.09 \pm 0.29$ & $3.58 \pm 0.21$ \\
\hline Kidney & $0.59 \pm 0.29$ & $0.66 \pm 0.11$ & $0.56 \pm 0.11$ & $1.40 \pm 0.31$ & $1.40 \pm 0.21$ & $1.98 \pm 0.17$ \\
\hline Intestine & $0.71 \pm 0.19$ & $0.82 \pm 0.12$ & $0.84 \pm 0.10$ & $2.25 \pm 0.23$ & $2.69 \pm 0.37$ & $2.87 \pm 0.14$ \\
\hline Bladder & $1.60 \pm 0.21$ & $1.17 \pm 0.17$ & $2.05 \pm 0.27$ & $6.51 \pm 0.58$ & $5.66 \pm 0.41$ & $6.45 \pm 0.57$ \\
\hline Muscle & $5.00 \pm 0.57$ & $6.79 \pm 0.69$ & $10.58 \pm 0.67$ & $9.64 \pm 0.63$ & $13.67 \pm 1.04$ & $7.17 \pm 0.83$ \\
\hline Skin & $3.08 \pm 0.23$ & $1.42 \pm 0.12$ & $1.65 \pm 0.12$ & $1.77 \pm 0.09$ & $2.22 \pm 0.19$ & $3.00 \pm 0.36$ \\
\hline
\end{tabular}

Tumor to normal tissue ratios (mean and standard deviation, $\mathrm{n}=6$ ) were calculated by dividing the fluorescence intensity $/ \mathrm{mg}$ (e.e/mg) of the tumor by the e.e/mg of the different normal tissues indicated. Ps A, photosensitizer A; Ps I, photosensitizer I.

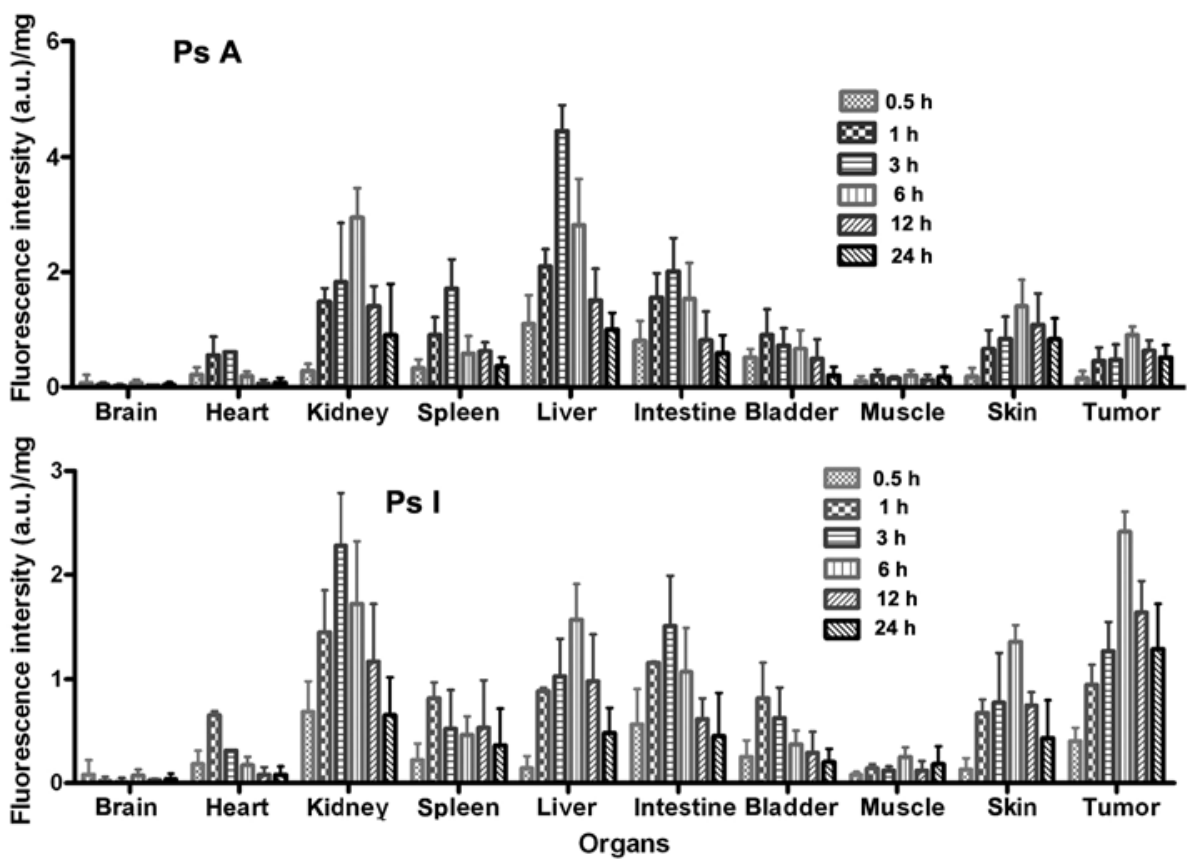

Figure 4. Comparison of the biodistribution of photosensitizer I (Ps I) in tumor and normal tissues of various organs in nude mice using a chemical extraction method. Each data point is the mean of 6 animals \pm standard deviation (SD).

and the lowest amounts were found in the brain for tumor and normal tissues (Fig. 4). However, the amount of Ps I accumulation was clearly reduced in the normal tissues as compared to that of Ps A at all time-points. By contrast, as early as $0.5 \mathrm{~h}$ after injection, Ps I exhibited, on average, a 2.7-fold higher tumor uptake than Ps A, and the increased tumor uptake persisted. As a consequence, the tumor to normal tissue ratios were $>2$-fold higher for Ps I than for Ps A at all the tested time-points (Table I).

PDT efficacy of Ps I in vivo. The antitumor efficiency of Ps I-PDT against xenograft tumors in vivo is shown in 


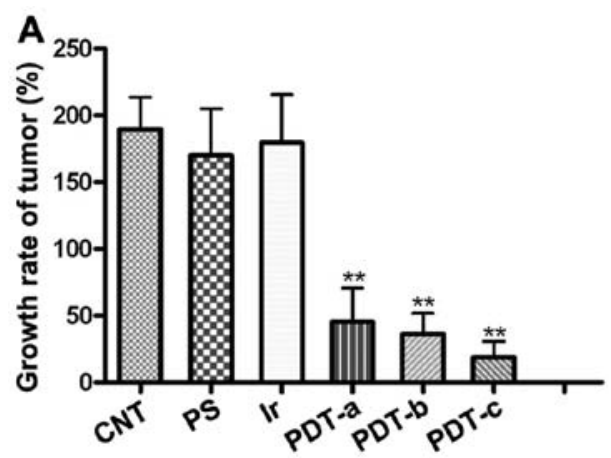

B

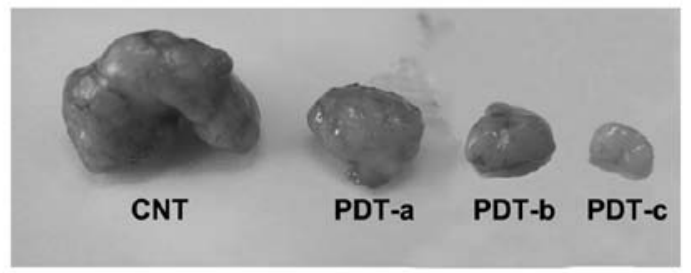

Figure 5. Effect of photosensitizer I (Ps I)-photodynamic therapy (PDT) on the tumor growth. (A) Tumor growth rate. (B) Images of tumors excised on day 14. CNT, untreated nude mice; Ps, only injected with $5 \mathrm{mg} / \mathrm{kg}$ of Ps I; Ir, only treated with $80 \mathrm{~J} / \mathrm{cm}^{2}$ of red light; PDT-a, after injection with $5 \mathrm{mg} / \mathrm{kg}$ of Ps I, irradiated with $80 \mathrm{~J} / \mathrm{cm}^{2}$ of red light; PDT-b, after injection with $5 \mathrm{mg} / \mathrm{kg}$ of Ps I, irradiated with $100 \mathrm{~J} / \mathrm{cm}^{2}$ of red light; PDT-c: after injection with $7 \mathrm{mg} / \mathrm{kg}$ of Ps I, irradiated with $80 \mathrm{~J} / \mathrm{cm}^{2}$ of red light.

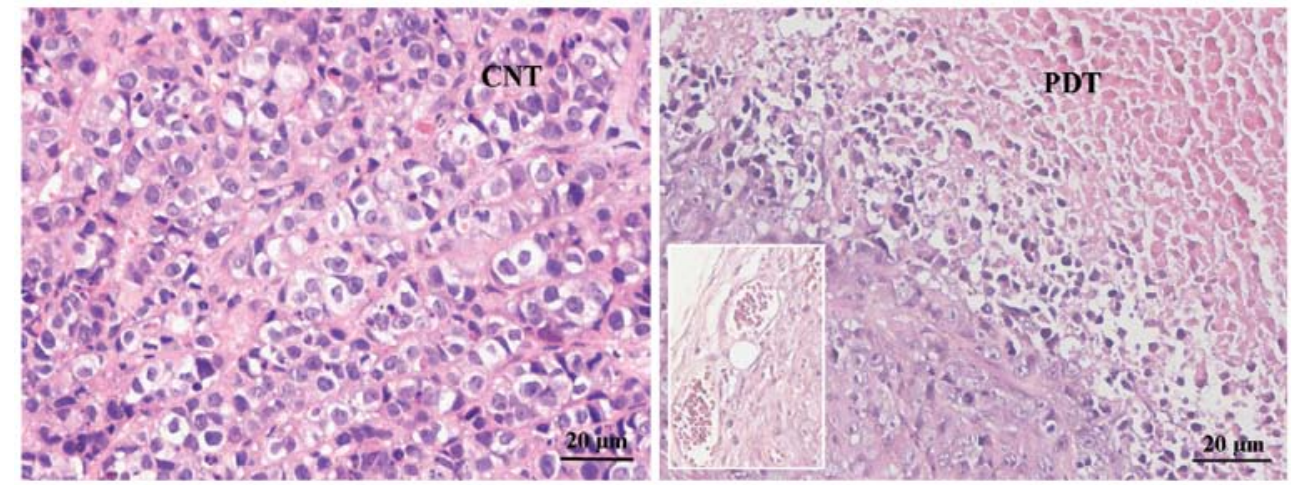

Figure 6. Microscopic images of the human cervical carcinoma xenograft nude tumors obtained at 14 day post-photodynamic therapy (PDT) (hematoxylin and eosin staining). CNT, untreated nude mice; PDT, after injection with $5 \mathrm{mg} / \mathrm{kg}$ of photosensitizer I (Ps I) at $6 \mathrm{~h}$, irradiated with $80 \mathrm{~J} / \mathrm{cm}^{2}$ of red light. Bar, $20 \mu \mathrm{m}$.

Fig. 5. In the negative control groups, no treatment (CNT), the light alone and Ps I alone, the tumor rapidly grew, while no significant difference among these groups was observed (Fig. 5A) $(\mathrm{P}>0.05)$. Of note, the Ps I-PDT was found to cause strong suppression of tumor growth, and the inhibition of Ps I-PDT was dependent on the concentration of Ps I and irradiation dose. The tumor volume in the control groups was much larger than that in the PDT groups $(\mathrm{P}<0.001)$, and the smallest tumor was found in the Ps I-PDT group treated with $7 \mathrm{mg} / \mathrm{kg}$ of Ps I and $80 \mathrm{~J} / \mathrm{cm}^{2}$ of irradiation. On the 14th day, the tumor volume in the CNT group was 4- and 10-fold larger than that of the PDT groups treated with 5 and $7 \mathrm{mg} / \mathrm{kg}$ of Ps I, respectively. The large area of amorphous necrosis and disorganization of tumor cells, reduction in the size and number of cell nuclei, fusion of cytoplasm, and congestion of blood vessels (inset) were evident in the Ps I-PDT group, although almost no effects were observed in the control groups (Fig. 6). However, the cell damage caused by PDT was not observed in the liver, spleen or kidney (data not shown).

Apoptosis of tumor cells. The TUNEL-positive apoptotic cells (green) in tumor sections were significantly increased in the Ps I-PDT group, whereas no significant TUNEL fluorescence was observed in the three control groups (Fig. 7A). The nuclear fragmentation and the apoptotic bodies were observed in the
Ps I-PDT group under electron microscopy (Fig. 7B and C). These results strongly suggested that cell apoptosis was only stimulated by Ps I-PDT, and this apoptosis inhibited tumor growth in the HeLa xenograft animal model.

\section{Discussion}

Cervical cancer is a considerable threat to public health. However, the currently available therapies are a combination of pelvic radiotherapy, radical surgery, and chemotherapy (21), which inevitably damage the surrounding normal tissues when destroying the tumors. As a conservative therapy, PDT can preserve complete organic structure for the patients and be repeated numerous times (22). Given the positive expression of the folate receptor on human cervical carcinoma HeLa cells (23) and the strong demand for conservative therapy methods to treat cervical carcinoma, HeLa cells and nude mice-bearing human cervical carcinoma xenografts were used as experimental models to evaluate the anticancer efficiency of Ps I-PDT in the present experimental study.

As Ps I is intravenously administered and is supposed to localize and kill only the malignant cells after light irradiation, the tumor targeting of Ps I is extremely important for the application of Ps I-PDT in cancer. Thus, the tumor targeting of Ps I was first analyzed in vitro and in vivo. The results in vitro 

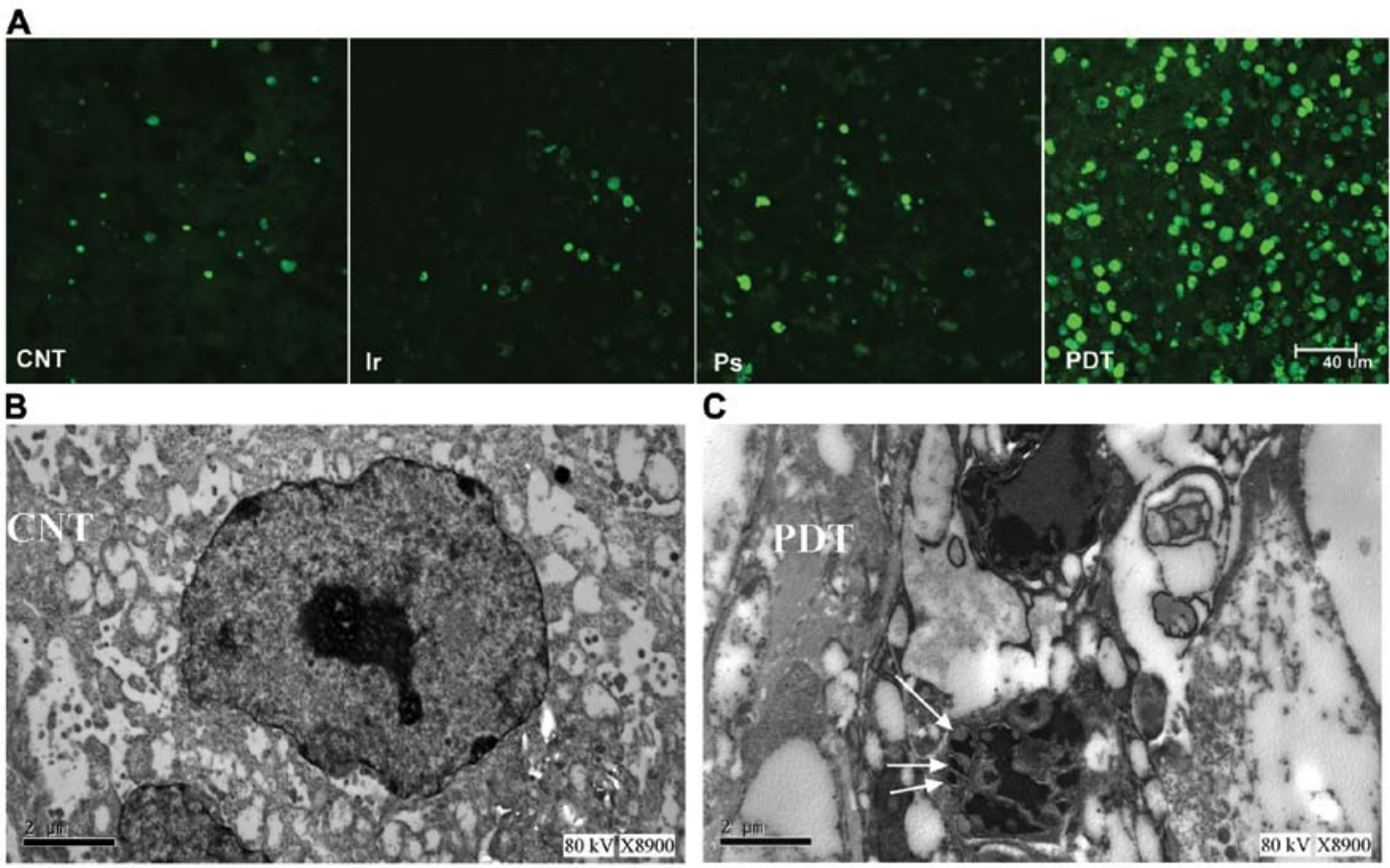

Figure 7. Apoptotic analysis in vivo. (A) The results of terminal deoxynucleotidyl transferase-mediated deoxyuridinetriphosphate nick end-labeling (TUNEL) assay observed under confocal microscopy. CNT, untreated nude mice; Ir, only treated with $80 \mathrm{~J} / \mathrm{cm}^{2}$ of red light; photosensitizer (Ps), only injected with $5 \mathrm{mg} / \mathrm{kg}$ of Ps I; photodynamic therapy (PDT), after injection with $5 \mathrm{mg} / \mathrm{kg}$ of Ps I at $6 \mathrm{~h}$, irradiated with $80 \mathrm{~J} / \mathrm{cm}^{2}$ of red light; Bar, $40 \mu \mathrm{m}$. (B and C) Electron micrographs of tumor in PDT group. Bar, $5 \mu \mathrm{m}$ in (B), $2 \mu \mathrm{m}$ in (C).

indicated that there was no obvious difference in the cellular uptake of Ps A with or without folic acid present. By contrast, the cellular uptake of Ps I by HeLa cells was much higher than that of Ps A, and the cellular uptake of Ps I was markedly inhibited by the presence of excess free folic acid, suggesting that the free folic acid molecules inhibited the cellular uptake of Ps I by competitively binding with the folate receptors on the surface of HeLa cells. In vivo, the tumor uptake of Ps I was enhanced, and the tumor to normal tissue ratio was considerably increased compared with Ps A. The results showed that as compared with Ps A, with the increased accumulation of Ps I in the tumor and the decrease in the skin, the ratio of tumor to skin for Ps I significantly increased. For example, the ratio of tumor to skin for Ps I was 2.72- and 4.91-fold to Ps A at 6 and $24 \mathrm{~h}$ post-injection, respectively. This finding was crucial for the reduction of cutaneous photosensitivity. However, we do not have a reasonable explanation at this time for the tumor targeting of Ps I being more significant in vitro than in vivo, and this finding requires further investigation.

Westermann et al (24) reported that the tumor selectivity of chlorin could be improved by conjugation with such a macromolecule as PEG because of the extended circulation half-life. To determine whether PEG improves the tumor targeting of Ps I and A, we compared the cellular uptake of Ps I and A by HeLa cells with their precursor, 5, 10, 15-tris (3-hydroxyphenyl)-20-(4-carboxyphenyl) chlorin. However, as the precursor is only soluble in DMSO and due to the membrane penetrant-carrier properties of DMSO (18), the cellular uptake of the precursor was higher than that of Ps I (data not shown).

The largest accumulation of Ps I in normal tissues was found in the kidney and not in the liver. This observation is not in agreement with the known tendency of most Ps used in the clinic, which preferentially accumulate in the liver, similar to Ps A (25). This may be due to the expression of the folate receptor on the apical membranes of kidney proximal tubule cells (26) resulting in more Ps I being linked to the kidney prior to excretion and then transcellularly returned back into circulation.

The antitumor activity of Ps I was first evaluated by MTT assay in vitro. Compared with Ps A, Ps I exhibited a much higher proliferation inhibition rate for HeLa cells, and the higher the concentration of the photosensitizer, the more obvious was the difference (Fig. 2A). For example, when the concentration of the photosensitizer increased from 3.8 to $60.8 \mu \mathrm{mol} / 1$, the difference in the inhibition rate between Ps I and $\mathrm{A}$ increased from 7.1 to $34.0 \%$. Given the much higher cellular uptake of HeLa cells for Ps I than for Ps A, the result may be explained. The phototoxicity of Ps I was then examined in vivo using human cervical tumor-bearing mice. Based on our results of the metabolism of Ps I, phototherapy was performed $6 \mathrm{~h}$ after the intravenous (i.v.) injection of Ps I (PDT groups) and saline (irradiation control groups). Ps I-PDT significantly inhibited tumor growth without causing any side effects to the mice post-PDT. Tumor growth inhibition in vitro and in vivo exhibited an obvious dependency on the photosensitizer concentration and light energy intensity.

Apoptosis is of particular importance in cancer treatment. To investigate the death mode of tumor cells induced by Ps I-PDT in cervical carcinoma, cell apoptosis was analyzed in vitro and in vivo. The exposure of plasma membrane phosphatidylserine is an early marker of apoptosis and can be detected by Annexin V staining. Accordingly, the percentage of apoptosis of HeLa cells post-PDT in vitro was analyzed first 
by flow cytometry with Annexin V and PI double-staining. Compared with the control groups, the number of apoptotic cells in Ps I-PDT group clearly increased, and the HeLa cell apoptosis displayed an obvious dependency on Ps I concentration and light energy intensity (data not shown). Given that the upper left quadrant represents cell fragments (27), the presence and increase in the cell fragment population in the upper left quadrant indicated that the Ps I-mediated PDT was able to damage the target cells. The apoptosis of HeLa cells post-Ps I-PDT was determined by the characteristic nuclear fragmentation and the representative apoptotic bodies observed with confocal and electron microscopy, respectively. Moreover, cell apoptosis was demonstrated in vivo by TUNEL assay and electron microscopy observation. These results confirm that Ps I-PDT induced HeLa cell death through apoptosis. However, the results of the histological examination revealed the congestion of blood vessels. Therefore, the Ps I-PDT antitumor effect may also cause vascular impairment, resulting in a deficient nutrient supply, which directly induces tumor cell apoptosis.

Taken together, the findings reported in the present study have demonstrated that Ps I shows higher targeting for HeLa cells and xenograft cervical carcinoma because of the special binding between folic acid with folate receptor and the endocytosis mediated by the folate receptor. As a consequence, Ps I-PDT exhibited much higher antitumor efficiency against the proliferation of HeLa cells and the growth of xenograft tumors. Apoptosis is therefore the main mode of HeLa cell death induced by Ps I-PDT.

\section{Acknowledgements}

The authors are grateful for the financial support from the National Nature Science Foundation of China (project no. 21072227).

\section{References}

1. Downs LS, Smith JS, Scarinci I, Flowers L and Parham G: The disparity of cervical cancer in diverse populations. Gynecol Oncol 109: 22-30, 2008.

2. Wells SF: Cervical cancer: an overview with suggested practice and policy goals. Medsurg Nurs 17: 43-50, 2008

3. He GF, Bian ML, Zhao YW, Xiang Q, Li HY and Xiao C: A study on the mechanism of 5-aminolevulinic acid photodynamic therapy in vitro and in vivo in cervical cancer. Oncol Rep 21 : $861-868,2009$.

4. Fayter D, Corbett M, Heirs M, Fox D and Eastwood A: A systematic review of photodynamic therapy in the treatment of pre-cancerous skin conditions, Barrett's oesophagus and cancers of the biliary tract, brain, head and neck, lung, oesophagus and skin. Health Technol Asses 14: 1-288, 2010.

5. Benov L: Photodynamic therapy: current status and future directions. Med Princ Pract: May 10, 2014 (Epub ahead of print) doi: 10.1159/000362416.

6. Syu WJ, Yu HP, Hsu CY, et al: Improved photodynamic cancer treatment by folate conjugated polymeric micelles in a KB xenografted animal model. Small 8: 2060-2069, 2012.

7. Dabrowski JM, Arnaut LG, Pereira MM, Monteiro CJ, Urbanska K, Simoes S and Stochel G: New halogenated water-soluble chlorin and bacteriochlorin as photostable PDT sensitizers: synthesis, spectroscopy, photophysics, and in vitro photosensitizing efficacy. ChemMedChem 5: 1770-1780, 2010.
8. Eshghi H, Sazgarnia MSc A, Rahimizadeh M, Attaran N Bakavoli $M$ and Soudmand S: Protoporphydin IX-gold nanoparticle conjugates as an efficient photosensitizer in cervial cancer therapy. Photodiagnosis Photodyn Ther 10: 304-312, 2013.

9. Shupin-Mrugalska P, Piskorz J, Goslinski T, Mielcarek J, Konopka K and Düzgüneş N: Current status of liposomal porphyrinoid photosensitizers. Drug Discov Today 18: 776-784, 2013.

10. Gijsens A, Missiaen L, Merlevede W and de Witte P: Epidermal growth factor-mediated targeting of chlorin e6 selectively potentiates its photodynamic activity. Cancer Res 60: 2197-2202, 2000.

11. Stefflova K, Li H, Chen J and Zheng G: Peptide-based pharmacomodulation of a cancer-targeted optical imaging and photodynamic therapy agent. Bioconjug Chem 18: 379-388, 2007.

12. Vrouenraets MB, Visser GWM, Loup C, et al: Targeting of a hydrophilic photosensitizer by use of internalizing monoclonal antibodies: A new possibility for use in photodynamic therapy. Int J Cancer 88: 108-114, 2000

13. McCarthy JR, Bhaumik J, Merbouh N and Weissleder R: High-yielding syntheses of hydrophilic, conjugatable chlorins and bacteriochlorins. Org Biomol Chem 7: 3430-3436, 2009.

14. Vlahov IR and Leamon CP: Engineering folate-drug conjugates to target cancer: from chemistry to clinic. Bioconjug Chem 23: 1357-1369, 2012.

15. Bugaj AM: Targeted photodynamic therapy - a promising strategy of tumor treatment. Photochem Photobiol Sci 10: 1097-1109, 2011.

16. Leung WN, Sun X, Mak NK and Yow CM: Photodynamic effects of mTHPC on human colon adenocarcinoma cells: photocytotoxicity, subcellular localization and apoptosis. Photochem Photobiol 75: 406-411, 2002.

17. Wang L, Li M and Zhang N: Folate-targeted docetaxel-lipidbased-nanosuspensions for active-targeted cancer therapy. Int J Nanomedicine 7: 3281-3294, 2012.

18. Li D, LI P, Lin H, Jiang Z, Guo L and Li B: A novel chlorin-PEGfolate conjugate with higher water solubility, lower cytotoxicity, better tumor targeting and photodynamic activity. J Photochem Photobiol B 127: 28-37, 2013.

19. Vaidya A, Sun Y, Feng Y, Emerson L, Jeong EK and Lu ZR: Contrast-enhanced MRI-guided photodynamic cancer therapy with a pegylated bifunctional polymer conjugate. Pharm Res 25 : 2002-2011, 2008.

20. Chin WWL, Heng PWS, Thong PSP, et al: Improved formulation of photosensitizer chlorin e6 polyvinylpyrrolidone for fluorescence diagnostic imaging and photodynamic therapy of human cancer, Eur J Pharm Biopharm 69: 1083-1093, 2008.

21. Gui T, Wang Y, Mao Y, Liu J, Sun S, Cao D, Yang J and Shen K: Comparisons of 5-aminolevulinic acid photodynamic therapy and after-loading radiotherapy in vivo in cervical cancer. Clin Transl Oncol 15: 434-442, 2013.

22. Hiorth M, Liereng L, Reinertsen R and Tho I: Formulation of bioadhesive hexylaminolevulinate pellets intended for photodynamic therapy in treatment of cervical cancer. Int J Pharm 441: 544-554, 2013.

23. Saul JM, Annapragada A, Natarajan JV and Bellamkonda RV: Controlled targeting of liposomal doxorubicin via the folate receptor in vitro. J Control Release 92: 49-67, 2003.

24. Westermann P, Glanzmann T, Andrejevic S, et al: Long circulating half-life and high tumor selectivity of the photosensitizer meta-tetrahydroxyphenylchlorin conjugated to polyethylene glycol in nude mice grafted with a human colon carcinoma. Int J Cancer 76: 842-850, 1998.

25. Evensen JF, Moan J, Hindar A and Sommer S: Tissue distribution of ${ }^{3} \mathrm{H}$-hematoporphyrin derivative and its main components, ${ }^{67} \mathrm{Ga}$ - and ${ }^{131} \mathrm{I}$-albumin in mice bearing Lewis lung carcinoma. Prog Clin Biol Res 170: 541-562, 1984.

26. Leamon CP and Reddy JA: Folate-targeted chemotherapy. Adv Drug Deliv Rev 56: 1127-1141, 2004.

27. Zhang JL, Deng L, Yao JZ, et al: Synthesis and photobiological study of a novel chlorin photosensitizer BCPD-18MA for photodynamic therapy. Bioorg Med Chem 19: 5520-5528, 2011. 